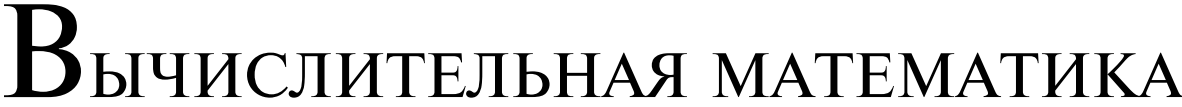

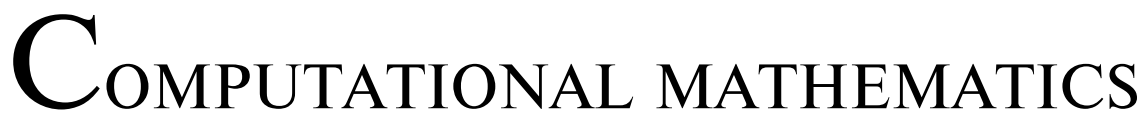

УДК 519.62

\section{СТАБИЛИЗИРОВАННЫЕ ЯВНЫЕ МЕТОАЫ ТИПА АААМСА}

\author{
В. И. РЕПНИКОВ ${ }^{1)}$ Б. В. ФАЛЕЙЧИК ${ }^{1)}$, А. В. МОЙСА ${ }^{1)}$ \\ ${ }^{1)}$ Белорусский государственный университет, пр. Независимости, 4, 220030, г. Минск, Беларусь
}

Представлены явные многошаговые методы типа Адамса с расширенным интервалом устойчивости, аналогичные явным стабилизированным чебышевским методам типа Рунге - Кутты. Доказано, что для любого $k \geq 1$ существует явный $k$-шаговый метод типа Адамса первого порядка с интервалом устойчивости длиной $2 k$. Коэффициенты и константа погрешности таких методов имеют весьма простой вид. Получена также демпфированная модификация этих методов. В общем случае для построения $k$-шагового метода порядка $p$ необходимо решить задачу условной оптимизации, в которой целевая функция и $p$ ограничений являются многочленами второй степени от $k$ переменных. Численно построены методы до шестого порядка включительно, проведены несколько вычислительных экспериментов для подтверждения свойств аппроксимации и устойчивости.

Ключевые слова: численное решение ОДУ; жесткость; интервал устойчивости; абсолютная устойчивость; многошаговые методы; методы типа Адамса; явные методы.

Благодарность. Работа выполнена при поддержке государственной программы научных исследований Республики Беларусь «Конвергенция-2020». Авторы также выражают благодарность рецензенту статьи за подробный и компетентный отзыв.

Образец цитирования:

Репников ВИ, Фалейчик БВ, Мойса АВ. Стабилизированные явные методы типа Адамса. Журнал Белорусского государственного университета. Математика. Информатика. 2021;2:82-98 (на англ.).

https://doi.org/10.33581/2520-6508-2021-2-82-98

\section{For citation:}

Repnikov VI, Faleichik BV, Moisa AV. Stabilised explicit Adamstype methods. Journal of the Belarusian State University. Mathematics and Informatics. 2021;2:82-98.

https://doi.org/10.33581/2520-6508-2021-2-82-98

\begin{abstract}
Авт оры:
Василий Иванович Репников - кандидат физико-математических наук; заведующий кафедрой вычислительной математики факультета прикладной математики и информатики. Борис Викторович Фалейчик - кандидат физико-математических наук; доцент кафедры вычислительной математики факультета прикладной математики и информатики. Андрей Владимирович Мойса - аспирант кафедры вычислительной математики факультета прикладной математики и информатики. Научный руководитель - Б. В. Фалейчик.
\end{abstract}

\footnotetext{
Authors:

Vasily I. Repnikov, $\mathrm{PhD}$ (physics and mathematics); head of the department of computational mathematics, faculty of applied mathematics and computer science.

repnikov@bsu.by

Boris V. Faleichik, $\mathrm{PhD}$ (physics and mathematics); associate professor at the department of computational mathematics, faculty of applied mathematics and computer science.

faleichik@bsu.by

Andrew V. Moisa, postgraduate student at the department of computational mathematics, faculty of applied mathematics and computer science.

moysa@bsu.by
} 


\title{
STABILISED EXPLICIT ADAMS-TYPE METHODS
}

\author{
V. I. REPNIKOV ${ }^{\mathrm{a}}$, B. V. FALEICHIK ${ }^{\mathrm{a}}, A . V . M O I S A^{\mathrm{a}}$ \\ ${ }^{a}$ Belarusian State University, 4 Niezaliežnasci Avenue, Minsk 220030, Belarus \\ Corresponding author: B. V. Faleichik (faleichik@bsu.by)
}

In this work we present explicit Adams-type multi-step methods with extended stability intervals, which are analogous to the stabilised Chebyshev Runge - Kutta methods. It is proved that for any $k \geq 1$ there exists an explicit $k$-step Adams-type method of order one with stability interval of length $2 k$. The first order methods have remarkably simple expressions for their coefficients and error constant. A damped modification of these methods is derived. In the general case, to construct a $k$-step method of order $p$ it is necessary to solve a constrained optimisation problem in which the objective function and $p$ constraints are second degree polynomials in $k$ variables. We calculate higher-order methods up to order six numerically and perform some numerical experiments to confirm the accuracy and stability of the methods.

Keywords: numerical ODE solution; stiffness; stability interval; absolute stability; multi-step methods; Adams-type methods; explicit methods.

Acknowledgements. The work is supported by Belarusian government program of scientific research «Convergence-2020». The authors also would like to thank the anonymous reviewer for valuable comments and suggestions.

\section{Introduction}

A $k$-step explicit Adams-type method for the numerical integration of the ordinary differential equation system

$$
y^{\prime}=f(t, y), y\left(t_{0}\right)=y_{0}, y: \mathbb{R} \rightarrow \mathbb{R}^{n}, f: \mathbb{R} \times \mathbb{R}^{n} \rightarrow \mathbb{R}^{n},
$$

on a uniform grid has the form

$$
y_{m+k}=y_{m+k-1}+\tau\left(\beta_{0} f_{m}+\ldots+\beta_{k-1} f_{m+k-1}\right),
$$

where $m$ is the step number, $y_{l} \approx y\left(t_{0}+l \tau\right), f_{l}=f\left(t_{0}+l \tau, y_{l}\right), l \geq 0, \tau$ is a discretisation step, and $\left\{\beta_{j}\right\}$, $j=0,1, \ldots, k-1$, are the coefficients for the method.

A conventional means of analysing the linear stability of a multi-step method is through the construction of a stability region $S \subset \mathbb{C}$ such that for all $\lambda \tau \in S$ the numerical solution of the model linear problem

$$
y^{\prime}=\lambda y, \lambda \in \mathbb{C},
$$

remains bounded for all $m$. The equivalent requirement is that all roots $\zeta_{j}$ of the characteristic equation

$$
\rho(\zeta)-\lambda \tau \sigma(\zeta)=0
$$

lie within the unit disc on the complex plane, and all the roots of modulus one are simple [1]. Here $\rho$ and $\sigma$ are the standard generating polynomials which in our case have the form

$$
\rho(\zeta)=\zeta^{k}-\zeta^{k-1}, \sigma(\zeta)=\sum_{j=0}^{k-1} \beta_{j} \zeta^{j}
$$

The stability interval of a method is the largest interval of the real axis of the form $[-\ell, 0]$ contained in $S$. Here $\ell \geq 0$ is the value which will be referred to as the length of the stability interval. As is known, stability intervals of the classical explicit Adams methods are small and get smaller with the growth of $k$, so these methods are not suitable for stiff problems. The purpose of the present research is to construct explicit multi-step methods of Adams type (1) of order $p<k$ with increased lengths of stability intervals. Putting it in other words, we develop a multi-step analog of the well-known Chebyshev Runge - Kutta methods [2-5].

The main obstacle for the way of construction of multi-step methods for stiff problems is the dependence of the error constant on the size of stability region, which was investigated by Jeltsch and Nevanlinna [6;7]. On the one hand, due to [5, theorem 4.2], for any $k>1, \alpha<\frac{\pi}{2}$ and $R>0$ there exists an explicit linear multi-step method of order $k-1$ such that the method is stable in the set

$$
\{\mu \in \mathbb{C}:|\mu| \leq R,|\arg (-\mu)| \leq \alpha\} .
$$

Unfortunately, methods which stability regions contain large disks of the form $\{\mu \in \mathbb{C}:|\mu+R| \leq R\}$ are useless in practice due to huge error constants (norms of Peano kernels) [6, theorem 4.1; 1, chapter V.2, theorem 2.6].

$$
\text { D2Y - cmovemtera ncmopura yonexa }
$$


On the other hand, in the case of long stability intervals the lower bounds for the error constant are less restrictive. Namely [6, theorem 4.4] gives a lower bound for Peano kernel norms for explicit multi-step methods in the form of $C_{k} \ell$, where $C_{k}>0$. Another interesting fact from [6, theorem 4.7] is that for explicit $k$-step methods of order $k-1$ with $\ell>2$ the error constant has lower bound equal to $\delta_{k-1} \frac{\ell-2}{2^{k-1}}$, where $\left(\delta_{k}\right)$ is a decreasing sequence with $\delta_{1}=0.5$. Thus, we hope that explicit multi-step methods with extended stability interval can have reasonable error constants (see section «Higher order methods»).

The material is organised as follows. In section «Optimisation strategy» we describe the general framework of the method's construction, sections «First order methods» and «First order methods with damping» are devoted to the first order methods and their damped modifications. Higher order methods are discussed in section «Higher order methods», and section «Numerical experiments» contains the results of numerical experiments. In the last section we discuss the obtained results and make final conclusions.

\section{Optimisation strategy}

The conventional way of constructing a stability region is to find a root locus curve $\mathcal{C}$ defined as

$$
\mathcal{C}=\left\{\mu_{\beta}\left(e^{i \varphi}\right): \varphi \in[0,2 \pi)\right\}
$$

where the function $\mu_{\beta}: \mathbb{C} \rightarrow \mathbb{C}$ maps a root of the characteristic equation (2) to the corresponding value of $\lambda \tau$ :

$$
\mu_{\beta}(\zeta)=\frac{\rho(\zeta)}{\sigma(\zeta)}
$$

The subscript $\beta$ indicates the dependence on the coefficients of method (1) which we are to be determined. From the definition of stability region it follows that $\partial S \subseteq \mathcal{C}$ and

thus the optimisation problem can be stated as

$$
\ell \leq \mu_{\beta}(-1),
$$

$$
\beta^{*}=\underset{\beta \in \mathcal{F} \cap \mathcal{P}}{\operatorname{argmin}} \mu_{\beta}(-1)
$$

where $\mathcal{P}$ is a set of coefficients which satisfy the posed order conditions, and $\mathcal{F}$ is a feasible set of coefficients with a desired shape of the root locus curve. This set is defined as follows.

Primarily we would like to have $\ell=-\mu_{\beta}(-1)$ for all $\beta \in \mathcal{F}$. To assure this we require the locus curve (3) not to cross the real axis before the parameter $\varphi$ reaches $\pi$. This condition triggers the following definition for the feasible set:

$$
\mathcal{F}=\left\{\beta \in \mathbb{R}^{k}: \operatorname{Im} \mu_{\beta}\left(e^{i \varphi}\right) \geq 0 \forall \varphi \in(0, \pi)\right\} .
$$

The main question now is how to find a parametrisation of $\mathcal{F}$ which allows the reduction of (5), (6) to some manageable form. We start by noting that

where

$$
\operatorname{Im} \mu_{\beta}\left(e^{i \varphi}\right) \geq 0 \Leftrightarrow v_{\beta}(\varphi) \geq 0,
$$

$$
v_{\beta}(\varphi)=\operatorname{Im} \rho\left(e^{i \varphi}\right) \overline{\sigma\left(e^{i \varphi}\right)}=\sum_{j=1}^{k}\left(\beta_{k-j}-\beta_{k-j-1}\right) \sin j \varphi .
$$

From here we set $\beta_{j}=0$ for all $j<0$ and $j>k-1$. By utilising the Chebyshev polynomials of the second kind $U_{j}$,

$$
U_{j-1}(\cos \varphi) \sin \varphi=\sin j \varphi,
$$

and using the power reduction formulae for the powers of $\cos \varphi,(7)$ can be represented as

$$
v_{\beta}(\varphi)=\sin \varphi \sum_{j=0}^{k-1} a_{j} \cos j \varphi
$$

with some $a_{j} \in \mathbb{R}$. Since we need $v_{\beta}(\varphi)$ to be non-negative on $(0, \pi)$, the following result from [7, lemma 6.1.3] is useful.

Lemma. For any non-negative trigonometric polynomial a of the form

$$
A(\varphi)=\sum_{j=0}^{k} a_{j} \cos j \varphi, a_{j} \in \mathbb{R},
$$


there exists a trigonometric polynomial

such that $A(\varphi)=|B(\varphi)|^{2}$.

$$
B(\varphi)=\sum_{j=0}^{k} b_{j} e^{i j \varphi}, b_{j} \in \mathbb{R}
$$

From this lemma it follows that all feasible trigonometric polynomials $v_{\beta}$ have the form

$$
v_{\beta}(\varphi)=\sin \varphi\left|\sum_{j=0}^{k-1} b_{j} e^{i j \varphi}\right|^{2}=\sin \varphi \sum_{j, l=0}^{k-1} b_{j} b_{l} \cos (j-l) \varphi
$$

with $b_{j} \in \mathbb{R}$. To complete the transformation of the optimisation problem we must express the original coefficients $\left\{\beta_{j}\right\}$ in terms of $\left\{b_{j}\right\}$. This can be done by converting (8) to the same basis of $\sin j \varphi$ as (7):

$$
\sin \varphi \sum_{j=0}^{k-1} a_{j} \cos j \varphi=\frac{1}{2} \sum_{j=0}^{k-1} a_{j}(\sin (j+1) \varphi-\sin (j-1) \varphi) .
$$

By equating this expression with (7) it is straightforward to get

$$
\begin{gathered}
\beta_{j}=\frac{1}{2}\left(\tilde{a}_{j-1}+\tilde{a}_{j}\right), j=0,1, \ldots, k-2, \\
\beta_{k-1}=\tilde{a}_{k-1}+\frac{\tilde{a}_{k-2}}{2} .
\end{gathered}
$$

Here for clarity $\tilde{a}_{j}=a_{k-1-j}, \tilde{a}_{-1}=0$. Conversely, from (8), (9) we have

$$
\begin{gathered}
\tilde{a}_{j}=2 \sum_{l=0}^{j} b_{l} b_{k-1+l-j}, j=0,1, \ldots, k-2, \\
\tilde{a}_{k-1}=\sum_{j=0}^{k-1} b_{j}^{2} .
\end{gathered}
$$

Transformations (10)-(10c) define the required mapping

where $b=\left(b_{0}, \ldots, b_{k-1}\right), \beta=\left(\beta_{0}, \ldots, \beta_{k-1}\right)$.

$$
T: b \rightarrow \beta
$$

Now let us derive the form of objective function (5),

in terms of $b$. By direct application of $(10)-(10 \mathrm{c})$ we have

$$
\mu_{\beta}(-1)=\frac{2 \cdot(-1)^{k}}{\sum_{j=0}^{k-1}(-1)^{j} \beta_{j}}
$$

$$
\sum_{j=0}^{k-1}(-1)^{j} \beta_{j}=(-1)^{k} \sum_{j=0}^{k-1} b_{j}^{2}
$$

so the initial optimisation problem $(5),(6)$ finally takes the surprisingly simple form

where $\mathcal{P}_{p}^{\prime}$ is the order $p$ restriction set:

$$
b^{*}=\underset{\beta \in \mathcal{P}_{p}^{\prime}}{\operatorname{argmin}} \sum_{j=0}^{k-1} b_{j}^{2}
$$

$$
\begin{gathered}
\mathcal{P}_{p}^{\prime}=\left\{b \in \mathbb{R}^{k}: G_{q}(T(b))=0, q=1,2, \ldots, p\right\}, \\
G_{1}(\beta)=\sum_{j=0}^{k-1} \beta_{j}-1, \\
G_{q}(\beta)=\sum_{j=0}^{k-1}(1-k+j)^{q-1} \beta_{j}-\frac{1}{q}, q>1 .
\end{gathered}
$$




\section{First order methods}

Theorem 1. For any number of steps $k>1$ there exists a first order explicit Adams-type method with stability interval of length $2 k$. The method has the form

i. e., $\beta_{j}=\frac{2 j+1}{k^{2}}$.

$$
y_{k+m}=y_{k+m-1}+\frac{\tau}{k^{2}}\left(f_{m}+3 f_{m+1}+\ldots+(2 k-1) f_{m+k-1}\right),
$$

P r o o f. Directly applying (10)-(10c) to the first order condition $(12 b)$ we have

$$
\beta_{0}+\beta_{1}+\ldots+\beta_{k-1}=a_{0}+a_{1}+\ldots+a_{k-1}=1 .
$$

Conversely, by the construction form (8), (9) we have

$$
\sum_{j=0}^{k-1} a_{j}=\sum_{j, l}^{k-1} b_{j} b_{l}=\left(\sum_{j=1}^{k-1} b_{j}\right)^{2} .
$$

Thus, the optimisation problem (12)-(12c) in the case of $p=1$ takes the form

subject to

$$
b^{*}=\underset{b \in \mathbb{R}^{k}}{\operatorname{argmin}}\left(b_{0}^{2}+b_{1}^{2}+\ldots+b_{k-1}^{2}\right)
$$

$$
\left(b_{0}+b_{1}+\ldots+b_{k-1}\right)^{2}=1 .
$$

Solving this problem by the method of Lagrange multipliers, we get $b_{j}^{*}=k^{-1}$ for all $j$ and then by (10)-(10c) obtain

$$
\beta^{*}=T\left(b^{*}\right)=\left(\frac{1}{k^{2}}, \frac{3}{k^{2}}, \ldots, \frac{2 k-1}{k^{2}}\right) .
$$

By construction of the method from (11) and since

we have $\ell=-\mu_{\beta^{*}}(-1)=2 k$.

$$
\sum_{j=0}^{k-1}(-1)^{j} \frac{2 j+1}{k^{2}}=(-1)^{k} \frac{1}{k},
$$

There is an interesting parity of the above result with the case of $s$-stage Chebyshev Runge - Kutta methods of order 1 , which require $s$ evaluations of $f$ per step and have stability interval equal to $2 s^{2}[1 ; 2]$. This allows us to suppose that the achieved length of $2 k$ is the largest possible for explicit first order multi-step methods.

Error constant. According to [9] we define the error constant of the multi-step method as

where

$$
C=\frac{C_{p+1}}{\sigma(1)}
$$

$$
C_{p+1}=\frac{1}{(p+1) !} \sum_{j=0}^{k}\left(a_{j} j^{p+1}-(p+1) \beta_{j} j^{p}\right) .
$$

It is easy to calculate this constant in our case.

Proposition 1. The error constant of the optimised first order methods is equal to

$$
C=\frac{k}{3}+\frac{1}{6 k}
$$

Pr o o f. Since $\beta_{j}=\frac{2 j+1}{k^{2}}, \alpha_{k}=1, \alpha_{k-1}=-1, \sigma(1)=1$, we have

$$
C=\frac{1}{2}\left(k^{2}-(k-1)^{2}-\frac{2}{k^{2}} \sum_{j=0}^{k} j(2 j+1)\right)=\frac{k}{3}+\frac{1}{6 k} \text {. }
$$

\section{First order methods with damping}

Analogously to the Chebyshev Runge - Kutta methods, in order to pull the root locus curve away from the real axis for $\varphi \in(0, \pi)$ it is necessary to perform a damping transformation with the constructed methods. 
Using (7), (8) consider (4) and represent

$$
\operatorname{Im} \mu_{\beta}\left(e^{i \varphi}\right)=Q(\varphi) \sin \varphi
$$

where

and

$$
Q(\varphi)=\frac{\sum_{j=0}^{k-1} a_{j} \cos j \varphi}{\left|\sigma\left(e^{i \varphi}\right)\right|^{2}}=\frac{\sum_{j=0}^{k-1} a_{j} \cos j \varphi}{\sum_{j=0}^{k-1} \delta_{j} \cos j \varphi}
$$

$$
\delta_{0}=\sum_{j=0}^{k-1} \beta_{j}^{2}, \delta_{j}=2 \sum_{l=0}^{j} \beta_{l} \beta_{j+l} .
$$

Recall that the connection between coefficients $a_{j}$ and $\beta_{j}$ is described by the first two equalities of (10)-(10c).

Let $\hat{Q}(\varphi)$ be the damped method's counterpart of (15). We define it as

$$
\hat{Q}(\varphi)=\frac{\sum_{j=0}^{k-1} \hat{a}_{j} \cos j \varphi}{\sum_{j=0}^{k-1} \hat{\delta}_{j} \cos j \varphi}=C(Q(\varphi)+\varepsilon),
$$

where $\varepsilon$ controls the «shift» from the real axis and $C$ is a scaling constant to be determined. Then we have $\hat{a}_{j}=C a_{j}^{\prime}$,

$$
a_{j}^{\prime}=a_{j}+\varepsilon \delta_{j} .
$$

Now we use this equality together with (10)-(10c) and get

$$
\begin{gathered}
\beta_{j}^{\prime}=\beta_{j}+\frac{\varepsilon}{2}\left(\delta_{k-j}+\delta_{k-j-1}\right), j=0,1, \ldots, k-2, \\
\beta_{k-1}^{\prime}=\beta_{k-1}+\frac{\varepsilon}{2} \delta_{1}+\varepsilon \delta_{0}, \delta_{k}=0 .
\end{gathered}
$$

The coefficients $\hat{\beta}_{j}$ of the sought damped method are expressed as $\hat{\beta}_{j}=C \beta_{j}^{\prime}$. To keep the order of the method equal to one, the constant $C$ should be equal to $\left(\sum_{j=0}^{k-1} \beta_{j}^{\prime}\right)^{-1}$. By (16) we have

$$
\sum_{j=0}^{k-1} \beta_{j}^{\prime}=\sum_{j=0}^{k-1} \beta_{j}+\varepsilon \sum_{j=0}^{k-1} \delta_{j}=\sum_{j=0}^{k-1} \beta_{j}+\varepsilon \sum_{j, l=0}^{k-1} \beta_{j} \beta_{l}
$$

Since $\sum_{j=0}^{k-1} \beta_{j}=1$ we finally obtain the following formulae for the coefficients of the damped method:

$$
\hat{\beta}_{j}=\frac{\beta_{j}+\varepsilon \Delta_{j}}{1+\varepsilon}, j=0,1, \ldots, k-1,
$$

where

$$
\begin{gathered}
\Delta_{j}=\frac{1}{2}\left(\delta_{k-j}+\delta_{k-j-1}\right), j=0,1, \ldots, k-2, \\
\Delta_{k-1}=\frac{1}{2} \delta_{1}+\delta_{0} .
\end{gathered}
$$

The values of $\Delta_{j}$ for $k$ from 2 to 10 for the optimised first order method (13) are presented in table 1 . The stability region boundaries of the one-step methods together with their damped counterparts are displayed in fig. 1. 

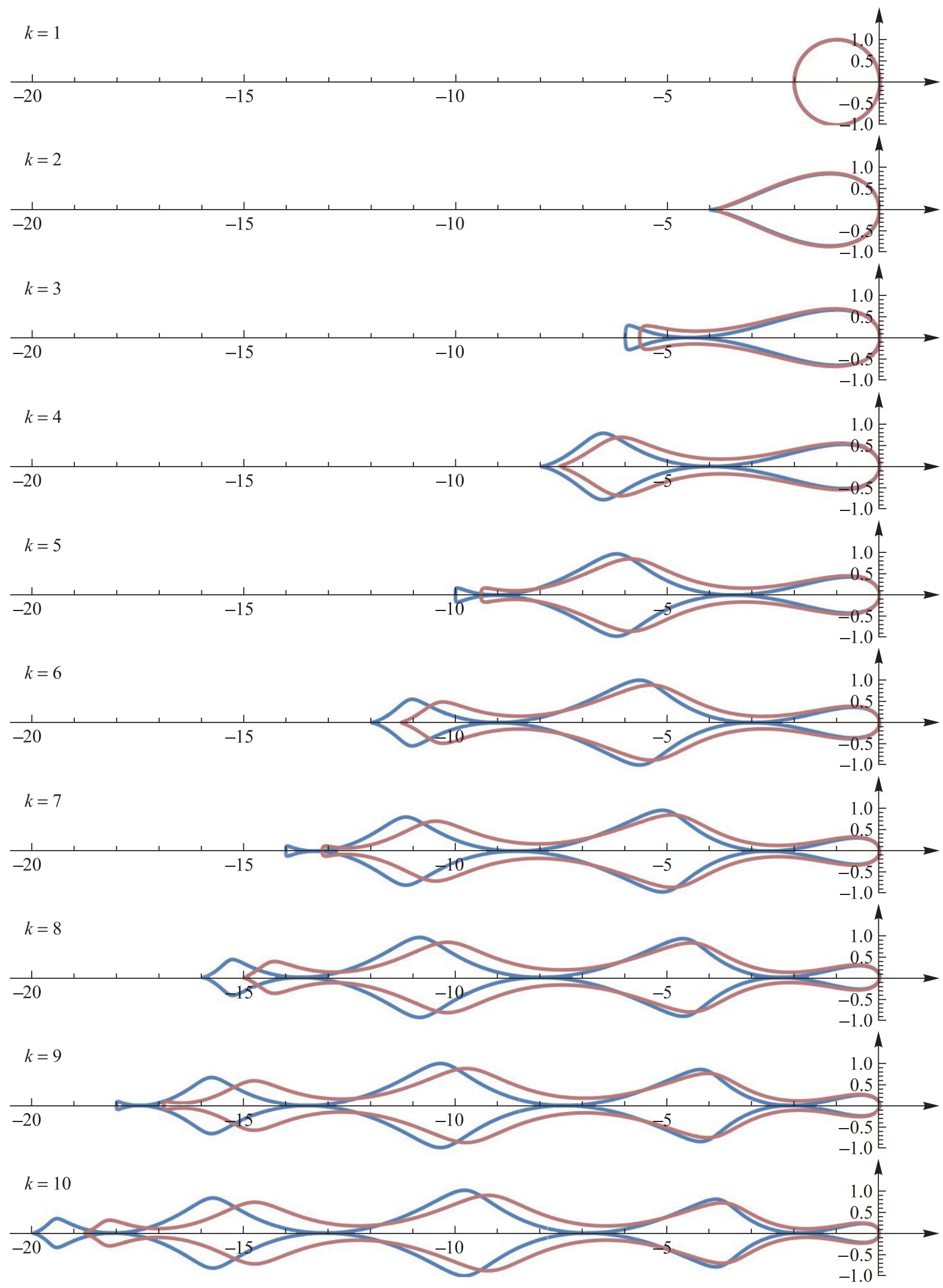

Fig. 1. Stability regions of the optimised first order methods and their damped versions $(\varepsilon=0.25)$ for $k=1, \ldots, 10$ 
The values of $\Delta_{j}$ for determining the coefficients of the

optimised first order methods with damping (17), $\beta_{j}=\frac{2 j+1}{k^{2}}$

\begin{tabular}{|c|c|c|c|c|c|c|c|c|c|c|}
\hline$k$ & $\Delta_{0}$ & $\Delta_{1}$ & $\Delta_{2}$ & $\Delta_{3}$ & $\Delta_{4}$ & $\Delta_{5}$ & $\Delta_{6}$ & $\Delta_{7}$ & $\Delta_{8}$ & $\Delta_{9}$ \\
\hline 2 & $\frac{3}{16}$ & $\frac{13}{16}$ & & & & & & & & \\
\hline 3 & $\frac{5}{81}$ & $\frac{23}{81}$ & $\frac{53}{81}$ & & & & & & & \\
\hline 4 & $\frac{7}{256}$ & $\frac{33}{256}$ & $\frac{79}{256}$ & $\frac{137}{256}$ & & & & & & \\
\hline 5 & $\frac{9}{625}$ & $\frac{43}{625}$ & $\frac{21}{625}$ & $\frac{187}{625}$ & $\frac{281}{625}$ & & & & & \\
\hline 6 & $\frac{11}{1296}$ & $\frac{53}{1296}$ & $\frac{131}{1296}$ & $\frac{79}{1296}$ & $\frac{121}{1296}$ & $\frac{167}{1296}$ & & & & \\
\hline 7 & $\frac{13}{2401}$ & $\frac{9}{343}$ & $\frac{157}{2401}$ & $\frac{41}{343}$ & $\frac{445}{2401}$ & $\frac{89}{343}$ & $\frac{813}{2401}$ & & & \\
\hline 8 & $\frac{15}{4096}$ & $\frac{73}{4096}$ & $\frac{183}{4096}$ & $\frac{337}{4096}$ & $\frac{527}{4096}$ & $\frac{745}{4096}$ & $\frac{983}{4096}$ & $\frac{1233}{4096}$ & & \\
\hline 9 & $\frac{17}{6561}$ & $\frac{83}{6561}$ & $\frac{209}{6561}$ & $\frac{43}{729}$ & $\frac{203}{2187}$ & $\frac{289}{2187}$ & $\frac{1153}{6561}$ & $\frac{1459}{6561}$ & $\frac{1777}{6561}$ & \\
\hline 10 & $\frac{19}{10000}$ & $\frac{93}{10000}$ & $\frac{47}{2000}$ & $\frac{437}{10000}$ & $\frac{691}{10000}$ & $\frac{989}{10000}$ & $\frac{1323}{10000}$ & $\frac{337}{2000}$ & $\frac{2067}{10000}$ & $\frac{2461}{10000}$ \\
\hline
\end{tabular}
Proposition 2. The stability interval of the damped method (17)-(17b) with $\beta_{j}=\beta_{j}^{*}=\frac{2 j+1}{k^{2}}$ is equal to
$\left[-\ell_{\varepsilon}, 0\right]$, where

Pro of. Let us compute

$$
\ell_{\varepsilon}=\frac{6(1+\varepsilon) k^{3}}{\varepsilon\left(4 k^{2}-1\right)+3 k^{2}}
$$

$$
\sum_{j=0}^{k-1}(-1)^{j} \hat{\beta}_{j}=(1+\varepsilon)^{-1}\left(\sum_{j=0}^{k-1}(-1)^{j} \beta_{j}^{*}+\varepsilon(-1)^{k-1} \delta_{0}^{*}\right) .
$$

The first term has already been calculated in (14) and the second is determined as

$$
\delta_{0}^{*}=\sum_{j=0}^{k-1}\left(\beta_{j}^{*}\right)^{2}=\sum_{j=0}^{k-1} \frac{(2 j+1)^{2}}{k^{4}}=\frac{4 k^{2}-1}{3 k^{3}} .
$$

From here we finally get

$$
\mu_{\hat{\beta}}(-1)=\frac{2(-1)^{k}}{\sum_{j=0}^{k-1}(-1)^{j} \hat{\beta}_{j}}=-\frac{6(1+\varepsilon) k^{3}}{\varepsilon\left(4 k^{2}-1\right)+3 k^{2}} .
$$

Corollary 1. The asymptotic length of the damped one-step method is

$$
\lim _{\varepsilon \rightarrow \infty} \ell_{\varepsilon}=\frac{3}{2} k
$$




\section{Higher order methods}

To construct a stabilised $k$-step Adams-type method of order $p$ one should use the general form of the optimisation problem (12)-(12c) with mapping $T$ specified by (10)-(10c). For example, for $k=5, p=4$, the problem in terms of $b_{j}$ takes the form

minimise

subject to

$$
b_{0}^{2}+b_{1}^{2}+b_{2}^{2}+b_{3}^{2}+b_{4}^{2}
$$

$$
\begin{aligned}
& \left(b_{0}+b_{1}+b_{2}+b_{3}+b_{4}\right)^{2}=1, \\
& b_{2} b_{3}+\left(3 b_{2}+b_{3}\right) b_{4}+b_{1}\left(b_{2}+3 b_{3}+5 b_{4}\right)+b_{0}\left(b_{1}+3 b_{2}+5 b_{3}+7 b_{4}\right)=-\frac{1}{2}, \\
& b_{2} b_{3}+\left(5 b_{2}+b_{3}\right) b_{4}+b_{1}\left(b_{2}+5 b_{3}+13 b_{4}\right)+b_{0}\left(b_{1}+5 b_{2}+13 b_{3}+25 b_{4}\right)=\frac{1}{3}, \\
& b_{2} b_{3}+\left(9 b_{2}+b_{3}\right) b_{4}+b_{1}\left(b_{2}+9 b_{3}+35 b_{4}\right)+b_{0}\left(b_{1}+9 b_{2}+35 b_{3}+91 b_{4}\right)=-\frac{1}{4} .
\end{aligned}
$$

The symbolic solution of this problem yielded by Wolfram Mathematica after transforming back to the initial variables $\beta_{j}$ is

$$
\beta^{*}=\left(-\frac{1}{4}, \frac{5}{8}, \frac{1}{24},-\frac{35}{24}, \frac{49}{24}\right)
$$

with $\ell=0.75$, to compare with $\ell=0.3$ for the classical explicit Adams-type method of order 4 . Another neat example is the 5-step method of order 2:

$$
\beta^{*}=\left(-\frac{1}{8}(3-\sqrt{5}),-\frac{3}{4}(\sqrt{5}-2), 0, \frac{7}{4}(\sqrt{5}-2), \frac{9}{8}(3-\sqrt{5})\right)
$$

with $\ell=2+\frac{4}{\sqrt{5}} \approx 3.789$. The stability regions of these and the rest of the 5 -step methods are shown in the uppermost part of fig. 2 .

Unfortunately, it is not always possible to obtain the solution symbolically, thus we compute the coefficients of our methods numerically using Mathematica's function NMinimise, see the corresponding code in Appendix A. We used 50-digit working precision and computed the $(k, p)$ methods for $k$ from 3 to 10 and $p$ from 2 to $k$ (with the latter value corresponding to the classical Adams methods). The results with 20-digit accuracy are displayed in tables 3 and 4 . It is interesting that the $(4,3)$ method coincides with $[9$, formula (5.4)]. The stability regions of 5-, 6- and 7-step methods are shown in fig. 2.

To assess the accuracy of the obtained coefficients we checked the magnitude of the order residuals $G_{q}\left(\beta^{*}\right)$ see (12b), (12c). In all convergent cases these residuals do not exceed $10^{-19}$. Note that Mathematica's function NMinimise failed to converge in the following cases: $(k, p)=(7,6)$ and for all $p>7$. Our hypothesis is that for these $(k, p)$ combinations the Adams-type methods satisfying our restrictions do not exist. Note that $(11,7)$ method seems to exist and has microscopic $\ell \approx 0.051$ which is just slightly more than $\ell=0.0465$ for the $(7,7)$ case. The error constants of all the calculated methods are presented in table 2 .

Error constants of the stabilised Adams-type methods

\begin{tabular}{|c|c|c|c|c|c|c|}
\hline$k$ & $p=1$ & $p=2$ & $p=3$ & $p=4$ & $p=5$ & $p=6$ \\
\hline 2 & 0.75 & & & & & \\
\hline 3 & 1.0556 & 0.66667 & & & & \\
\hline 4 & 1.375 & 1.0380 & 0.62500 & & & \\
\hline 5 & 1.7 & 1.5208 & 1.0227 & 0.59861 & & \\
\hline 6 & 2.0278 & 2.1128 & 1.5972 & 1.0120 & 0.57928 & \\
\hline 7 & 2.3571 & 2.8134 & 2.3814 & 1.6471 & 1.0032 & \\
\hline 8 & 2.6875 & 3.6223 & 3.4092 & 2.5751 & 1.6825 & 0.99505 \\
\hline 9 & 3.0185 & 4.5392 & 4.7148 & 3.8788 & 2.7235 & 1.7079 \\
\hline 10 & 3.35 & 5.5643 & 6.3328 & 5.6524 & 4.2616 & 2.8403 \\
\hline
\end{tabular}




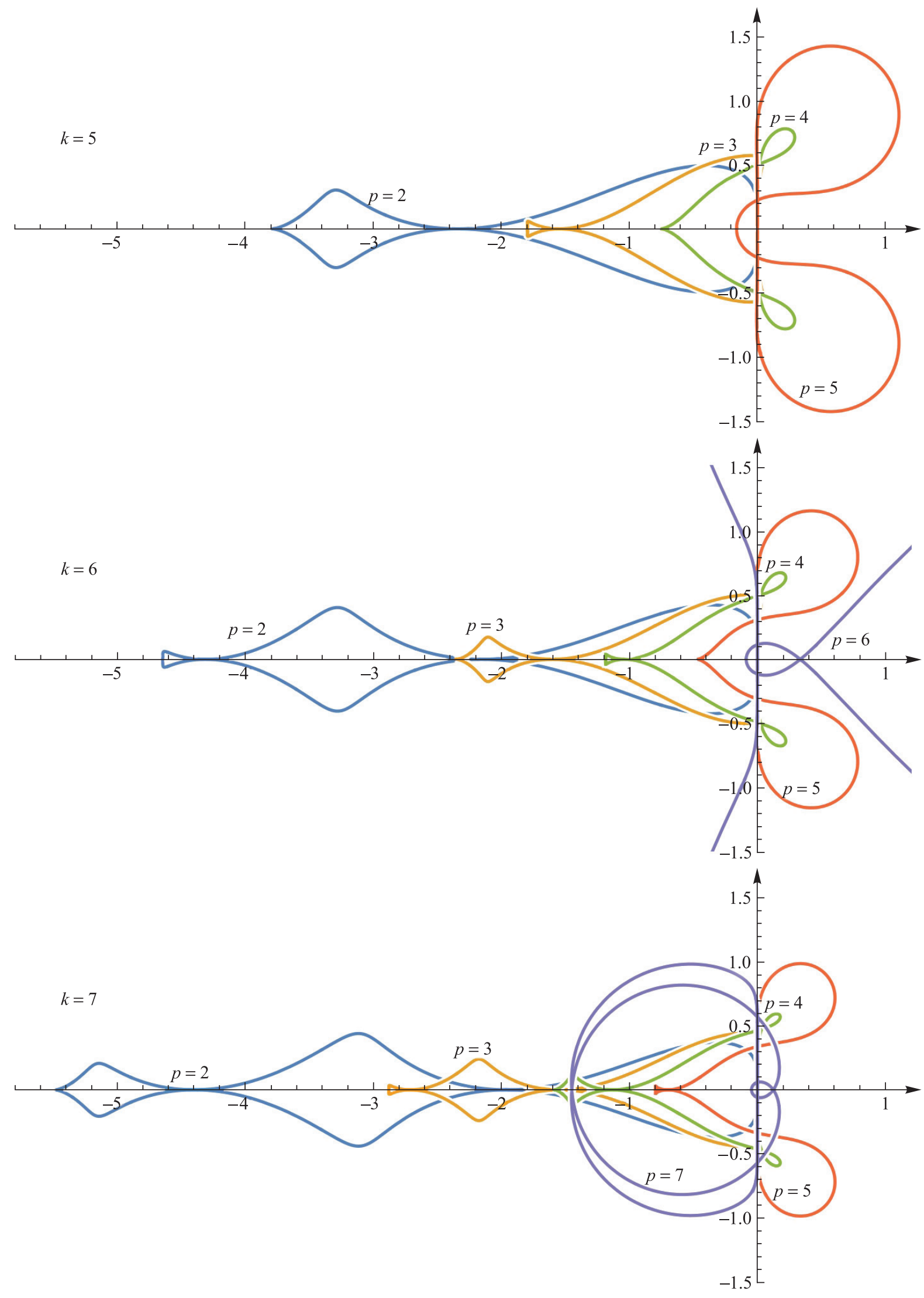

Fig. 2. Stability regions of the multi-step optimised methods for $k=5,6,7$ 
Coefficients and stability interval lengths

\begin{tabular}{|c|c|c|}
\hline$k$ & Order 2 & Order 3 \\
\hline 3 & $\begin{array}{l}\ell=2 \\
-0.25 \\
0 \\
1.25\end{array}$ & $\begin{array}{c}\ell=0.545454545454545455 \\
0.41666666666666666667 \\
-1.3333333333333333333 \\
1.9166666666666666667\end{array}$ \\
\hline 4 & $\begin{array}{c}\ell=2.914213562373095 \\
-0.14644660940673046069 \\
-0.18198051533945963691 \\
0.30330085889911065590 \\
1.0251262658470794417\end{array}$ & $\begin{array}{c}\ell=1.2 \\
0.25 \\
-0.33333333333333333333 \\
-0.58333333333333333333 \\
1.6666666666666666667\end{array}$ \\
\hline 5 & $\begin{array}{c}\ell=3.788854381999832 \\
-0.095491502812526287949 \\
-0.17705098312484227231 \\
0 \\
0.41311896062463196872 \\
0.85942352531273659154\end{array}$ & $\begin{array}{c}\ell=1.793779334348686 \\
0.16437694101246125619 \\
-0.0097910917750136439271 \\
-0.54022170408305993867 \\
-0.047691080558684215630 \\
1.4333269354042965420\end{array}$ \\
\hline 6 & $\begin{array}{c}\ell=4.642734410091836 \\
-0.066987298107786995665 \\
-0.14711431702997807715 \\
-0.089745962155603046598 \\
0.12564434701786943107 \\
0.44134295108991756459 \\
0.73686027918558112375\end{array}$ & $\begin{array}{c}\ell=2.347826086956522 \\
0.11574074074074731606 \\
0.087962962962956387640 \\
-0.28703703703705018768 \\
-0.40740740740739425676 \\
0.24537037037037694569 \\
1.2453703703703637950\end{array}$ \\
\hline 7 & $\begin{array}{c}\ell=5.484476959454063 \\
-0.049515566048790436882 \\
-0.11912520277278577227 \\
-0.11018250002552420585 \\
0 \\
0.19832850004594357054 \\
0.43679241016688116501 \\
0.64370235863427567947\end{array}$ & $\begin{array}{c}\ell=2.877558710633067 \\
0.085721156820309456282 \\
0.11154612811463327941 \\
-0.11721033134808636645 \\
-0.35463665779124584907 \\
-0.21744000532205222576 \\
0.39557372791516432979 \\
1.0964459816112773758\end{array}$ \\
\hline 8 & $\begin{array}{c}\ell=6.318535592272045 \\
-0.038060233744366798686 \\
-0.096797724520983369102 \\
-0.10779695287351088696 \\
-0.052994558379770972895 \\
0.068135860774038963863 \\
0.23715329632173532873 \\
0.41945680625751858277 \\
0.57090350616533915229\end{array}$ & $\begin{array}{c}\ell=3.391689975797208 \\
0.065966021983597280828 \\
0.11032441087323208003 \\
-0.022713554363876414313 \\
-0.23691021182637878968 \\
-0.30634225579338833174 \\
-0.055862376110155554778 \\
0.46825151960079988906 \\
0.97728644563616984059\end{array}$ \\
\hline 9 & $\begin{array}{c}\ell=7.147430550561413 \\
-0.030153689607037932268 \\
-0.079550128858107345641 \\
-0.098407115533249091604 \\
-0.073305865502781992742 \\
0 \\
0.11519493150433742625 \\
0.25585850038645603291 \\
0.39775064429061670700 \\
0.51261272331978661124 \\
\end{array}$ & $\begin{array}{c}\ell=3.895290219607647 \\
0.052301051895272605013 \\
0.10126696210118874790 \\
0.026642016446313140531 \\
-0.13793192915668298604 \\
-0.26695490513260400200 \\
-0.21907659037234928746 \\
0.064279256258874647289 \\
0.49902127636474858744 \\
0.88045286159523854733\end{array}$ \\
\hline 10 & $\begin{array}{c}\ell=7.972691637812280 \\
-0.024471741852422821505 \\
-0.066228831765768206903 \\
-0.087599164129385382526 \\
-0.078738975641538713579 \\
-0.034883488233566344682 \\
0.042635374507685291073 \\
0.14622952619142684103 \\
0.26279749238816316420 \\
0.37529671333936471557 \\
0.46496309519604145733\end{array}$ & $\begin{array}{c}\ell=4.391469108714782 \\
0.042467110956300544552 \\
0.090440497652067647206 \\
0.051030056647860180918 \\
-0.068250050077061163328 \\
-0.19902094851262917934 \\
-0.24395517042504782618 \\
-0.12913104538091815896 \\
0.14896994335213981908 \\
0.50694059780591167508 \\
0.80050900798137646097\end{array}$ \\
\hline
\end{tabular}


of the optimised methods of order $2-5$

\begin{tabular}{|c|c|}
\hline Order 4 & Order 5 \\
\hline $\begin{array}{c}\ell=0.3 \\
-0.37500000000000000000 \\
1.5416666666666666667 \\
-2.4583333333333333333 \\
2.2916666666666666667 \\
\end{array}$ & \\
\hline $\begin{array}{c}\ell=0.75 \\
-0.25 \\
0.625 \\
0.041666666666666666667 \\
-1.4583333333333333333 \\
2.0416666666666666667\end{array}$ & $\begin{array}{c}\ell=0.1633393829401088 \\
0.34861111111111111111 \\
-1.7694444444444444444 \\
3.6333333333333333333 \\
-3.8527777777777777778 \\
2.6402777777777777778\end{array}$ \\
\hline $\begin{array}{c}\ell=1.181897711989360 \\
-0.17622805914576966884 \\
0.21777962430380174276 \\
0.51616209424248971734 \\
-0.67621677042591625354 \\
-0.68603094336199527180 \\
1.8045340543873897341 \\
\end{array}$ & $\begin{array}{c}\ell=0.469157254561251 \\
0.24942129629629629629 \\
-0.89849537037037037036 \\
0.72476851851851851851 \\
1.1391203703703703704 \\
-2.6056712962962962963 \\
2.3908564814814814815\end{array}$ \\
\hline $\begin{array}{c}\ell=1.586803103995642 \\
-0.13027657069924974882 \\
0.040823321662060514133 \\
0.45157410201399110594 \\
0.016001789308425411316 \\
-0.79486796947441511195 \\
-0.18702302114721451156 \\
1.6037683483364023409\end{array}$ & $\begin{array}{c}\ell=0.792362028995767 \\
0.18480570522895041008 \\
-0.43546201769087620565 \\
-0.24616437886876401181 \\
1.2681635878048099022 \\
-0.32830322506067306370 \\
-1.5947509407373489642 \\
2.1517112693239019330 \\
\end{array}$ \\
\hline $\begin{array}{c}\ell=1.970916561391601 \\
-0.10001878254782277331 \\
-0.035748890463804216949 \\
0.30658371766113087497 \\
0.27749085554924180902 \\
-0.33516540035839458087 \\
-0.67199165170356770075 \\
0.12122231459728224806 \\
1.4376278372659343398 \\
\end{array}$ & $\begin{array}{c}\ell=1.105498503602666 \\
0.14160831078216433500 \\
-0.19477889703735130008 \\
-0.45247252238839228671 \\
0.57636630123759032103 \\
0.78354708230483585981 \\
-0.91953823465464150558 \\
-0.87725216386466696327 \\
1.9425201236204615397 \\
\end{array}$ \\
\hline $\begin{array}{c}\ell=2.339983407348191 \\
-0.079129092227346338565 \\
-0.067460438055823679907 \\
0.18522989963169925608 \\
0.31675641768693750027 \\
0.0076996887855987555993 \\
-0.48561642796053139031 \\
-0.48641107197220078201 \\
0.30896699066825414262 \\
1.2999640334434125362 \\
\end{array}$ & $\begin{array}{c}\ell=1.405151117615213 \\
0.11167958745225367479 \\
-0.068703909200215014827 \\
-0.41559134883278779976 \\
0.075957984853647800951 \\
0.78975711968445738645 \\
0.16879857406276817077 \\
-1.0382277451316307602 \\
-0.38771987715090903834 \\
1.7640496142624155802 \\
\end{array}$ \\
\hline $\begin{array}{c}\ell=2.698087099023256 \\
-0.064133502960306610717 \\
-0.078573353260495406661 \\
0.099782736471490155539 \\
0.27409149956975402355 \\
0.17521906381042658379 \\
-0.20265793719790100791 \\
-0.50346262595639964788 \\
-0.30713843196368842739 \\
0.42196137154381443077 \\
1.1849111799433059069\end{array}$ & $\begin{array}{c}\ell=1.692885048664239 \\
0.090219510737302839601 \\
-0.0021584562050617957037 \\
-0.32195487552605745395 \\
-0.17148478569282268595 \\
0.47486789482155684885 \\
0.59839764726184595395 \\
-0.27671853444446566397 \\
-0.94638400314820567730 \\
-0.057121557681252610888 \\
1.6123371598771602453\end{array}$ \\
\hline
\end{tabular}


Coefficients and stability interval lengths

\begin{tabular}{|c|c|c|}
\hline$k$ & Order 6 & Order 7 \\
\hline 6 & $\begin{array}{c}\ell=0.08771929824561404 \\
-0.3298611111111111111 \\
1.9979166666666666667 \\
-5.0680555555555555556 \\
6.9319444444444444444 \\
-5.5020833333333333333 \\
2.9701388888888888889\end{array}$ & \\
\hline 7 & NOT CONVERGED & $\begin{array}{c}\ell=0.04651391725937046 \\
0.31559193121693121693 \\
-2.2234126984126984127 \\
6.7317956349206349206 \\
-11.379894179894179894 \\
11.665823412698412698 \\
-7.3956349206349206349 \\
3.2857308201058201058\end{array}$ \\
\hline 8 & $\begin{array}{c}\ell=0.5290722934773335 \\
-0.19113689616832294585 \\
0.65850013289950086628 \\
-0.26698708897333444593 \\
-1.5041640716234265487 \\
1.8313158841283364334 \\
0.75394715979782998677 \\
-2.7632927648390354259 \\
2.4818176447784520799\end{array}$ & NOT CONVERGED \\
\hline 9 & $\begin{array}{c}\ell=0.7745044113664562 \\
-0.15072405770953055168 \\
0.36616417962483152368 \\
0.26486240742135927331 \\
-1.1679360960270178460 \\
-0.049706767276153478305 \\
1.8307408258122144817 \\
-0.54006451441787310785 \\
-1.8201171395869259716 \\
2.2667811621590956802\end{array}$ & NOT CONVERGED \\
\hline 10 & $\begin{array}{c}\ell=1.015322150308401 \\
-0.12149925981588955161 \\
0.19502001210515154522 \\
0.40323654967363550399 \\
-0.60200414081780015659 \\
-0.79801775043705878458 \\
0.91298862642764008111 \\
1.1648437230850238167 \\
-1.1001111732352200672 \\
-1.1334723376167517028 \\
2.0790157506312693158\end{array}$ & NOT CONVERGED \\
\hline
\end{tabular}


of the optimised methods of order 6-9

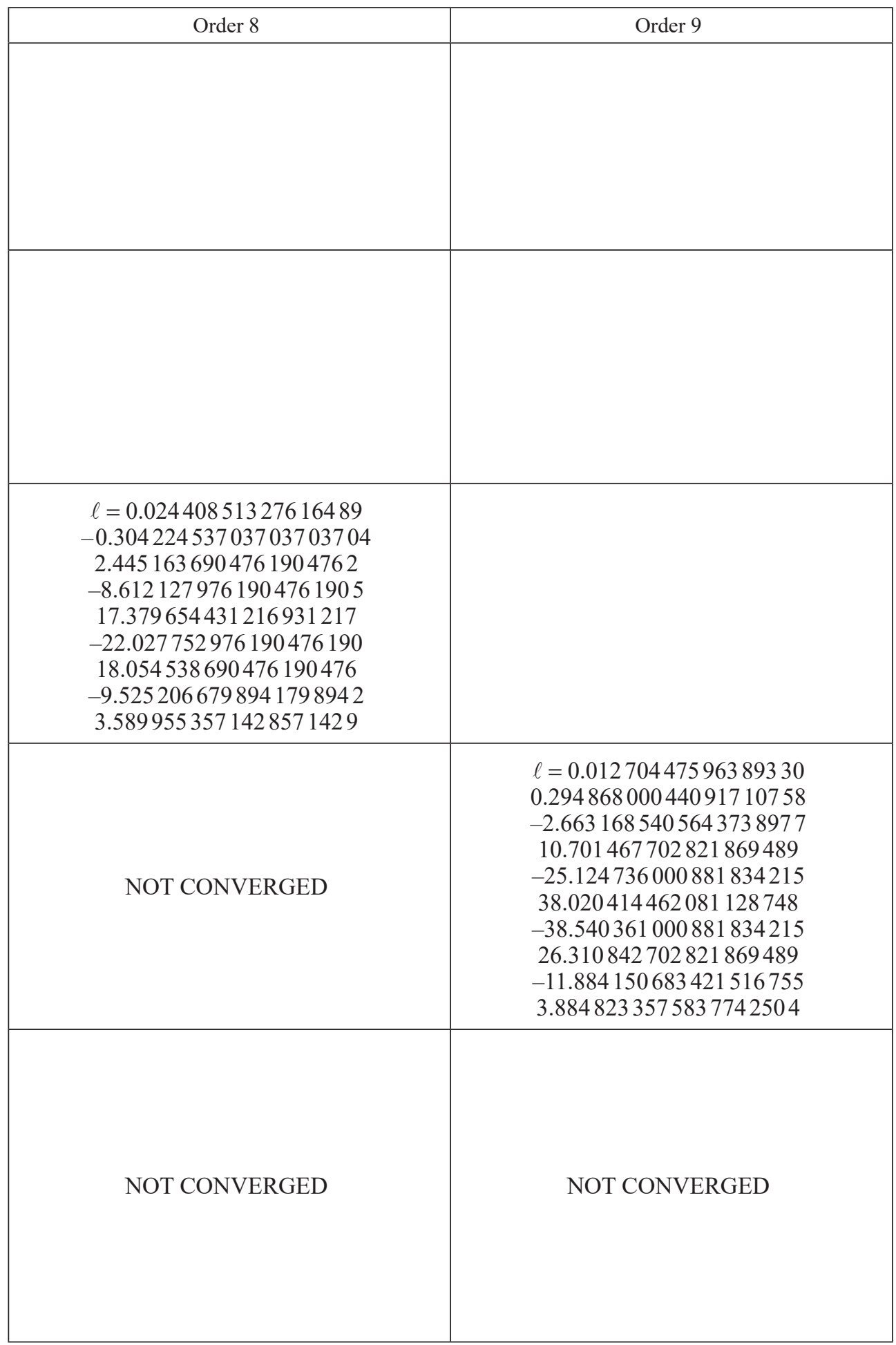




\section{Numerical experiments}

The purpose of the experiment is to verify accuracy and stability properties of the stabilised Adams-type methods constructed above. We also display results of the classic implicit Adams methods of corresponding orders, which have longer stability intervals than their classical explicit counterparts. In all our experiments we use constant step size and reference solutions computed by Wolfram Mathematica's NDSolve. The starting points were taken from this reference solution. For each method we perform a series of constant-step integrations with decreasing step size $\tau$ and calculate the maximum norm of the error at the endpoint. Missing points on the convergence diagrams mean that the error is too large due to instability of the method for the particular value of $\tau$.

HIRES. This is a classical mildly stiff test system of dimension 8 describing a chemical reaction (see [1, chapter IV.10, formula (10.4)]). All equations except the $6^{\text {th }}$ and $7^{\text {th }}$ are linear. The interval of integration is $[0,40]$. Figure $3, a$, shows the performance of the 6-step stabilised methods of orders $1-6$ and the implicit method of order 6 . We see that the results agree well with common sense: more accurate methods have shorter stability intervals. Then we compare methods of order 5 and display the results at fig. $3, b$, where we took $k$ from 9 to 15 in order to get larger stability intervals than the implicit method have. There is clear evidence that methods with larger $k$ have larger error constants. We do not show the results of the damped first order method, since the difference compared to the simple non-damped methods is negligible for this test problem.
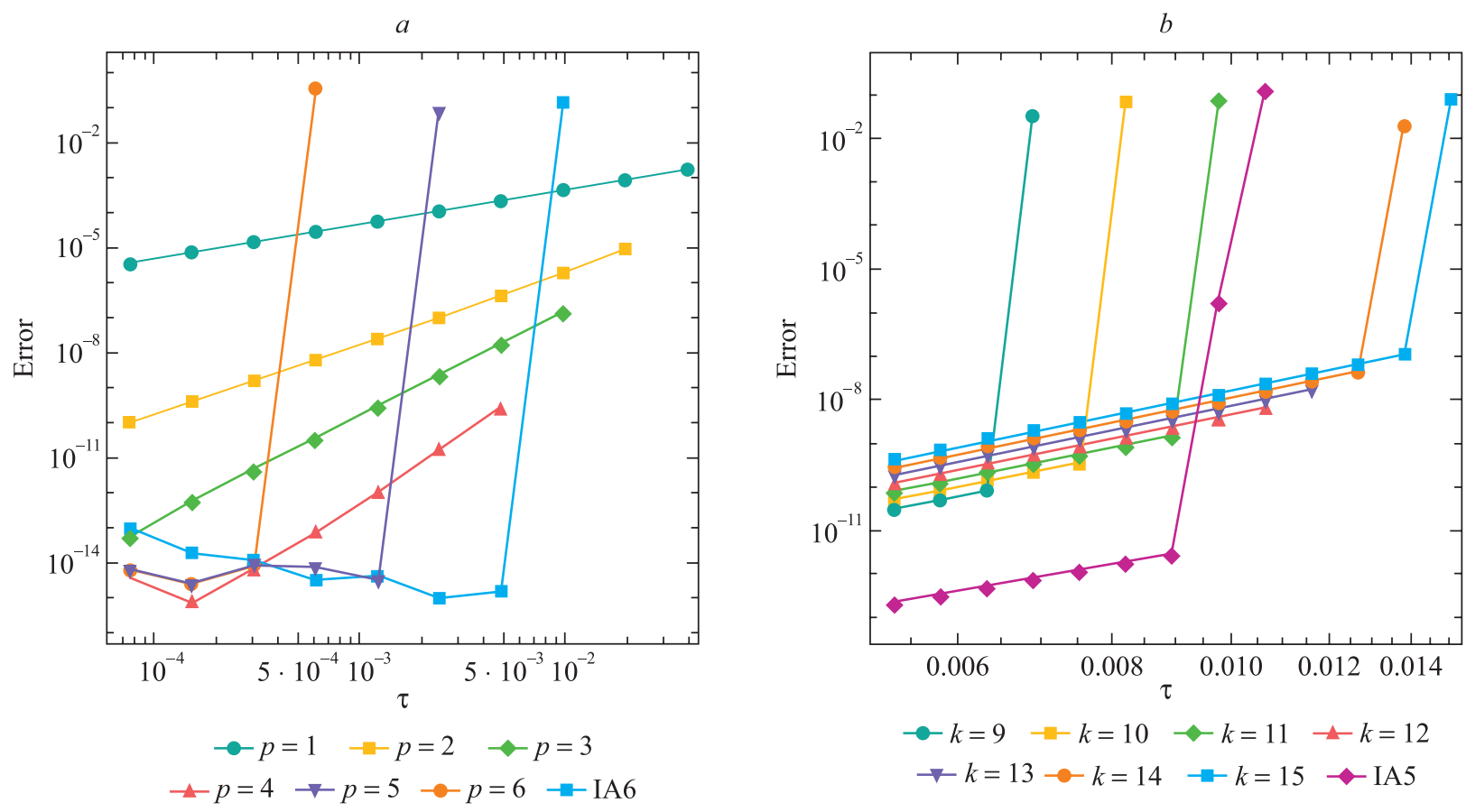

Fig. 3. Numerical experiment with HIRES problem.

Six-step stabilised methods and implicit Adams method of order $6(a)$.

Stabilised methods of order 5 and implicit Adams method of the same order $(b)$

Burgers' equation. The second problem is taken from [5]. Consider a method of lines discretisation of the one-dimensional non-linear boundary value problem

$$
\begin{gathered}
u_{t}+\left(\frac{u^{2}}{2}\right)_{x}=\mu u_{x x}, x \in[0,1], t \in[0,0.25], \\
u(x, 0)=1.5 x(1-x)^{2}, \\
u(0, t)=u(1, t)=0 .
\end{gathered}
$$

The spatial derivatives are approximated by standard central finite differences, the discretisation step is $\Delta x=\frac{1}{501}$, so the dimension of the resulting ordinary differential equation is 500 . The Jacobi matrix of this problem is not symmetric and complex eigenvalues occur for sufficiently small values of $\mu$. We took $\mu=0.005$ for which this is not the case at the starting point, but apparently non-real eigenvalues do emerge during integration. 
The first experiment is similar to the one from the previous problem. The results are presented on fig. 4, $a$ : we compare six-step methods of different orders. We see that the first order method with damping allows for taking longer time steps than the non-damped one. This indicates that the solution generates non-real eigenvalues of the Jacobi matrix. Hence, it is unlikely to benefit from using stabilized methods with large $k$ and $p$, for which we do not have damping yet.

$a$

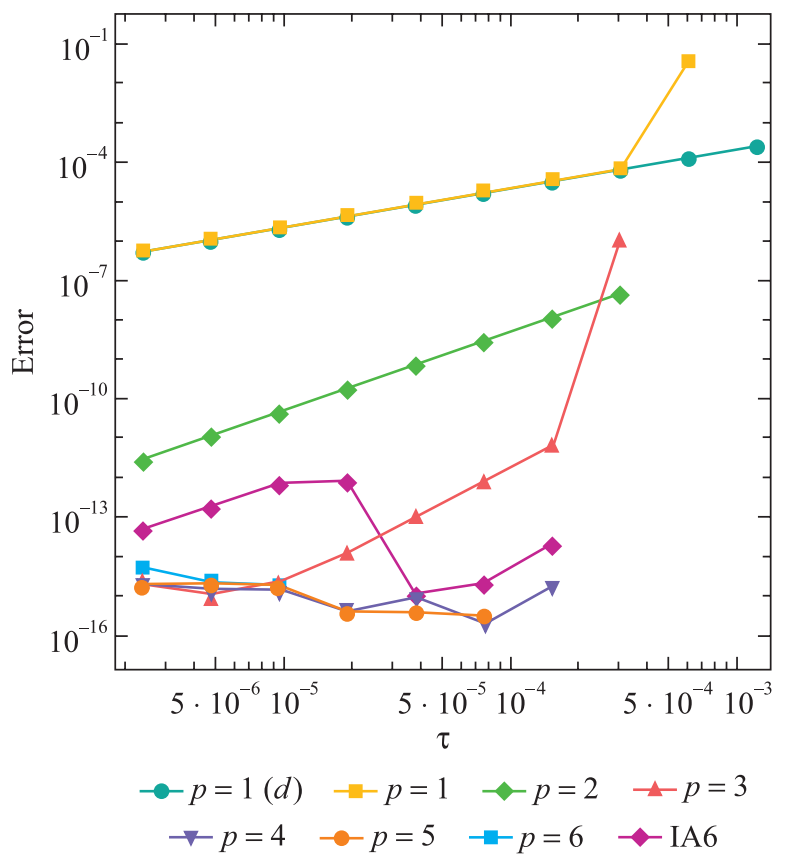

$b$

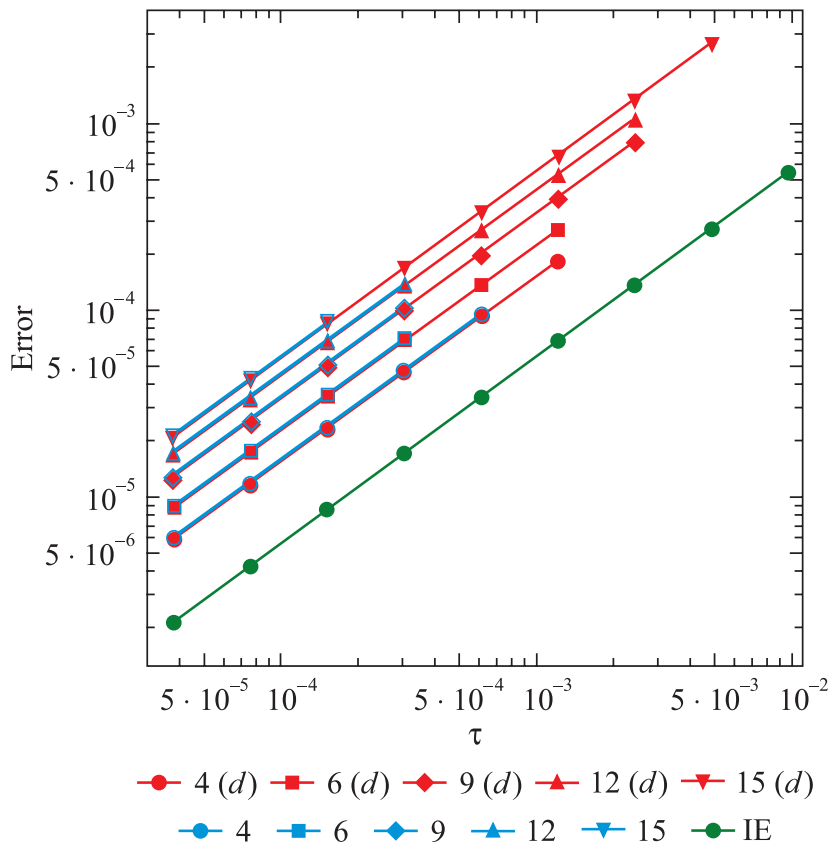

Fig. 4. Numerical experiment with Burgers' equation (18).

Six-step stabilised methods and implicit Adams method of order $6(a)$.

First order stabilised methods with and without damping

for $\varepsilon=0.25, k=4,6,9,12,15$, and the implicit Euler method $(b)$

Indeed, our experiments showed that these methods cannot take larger steps than implicit Adams-type methods of the same order, even if their stability interval is longer. Hence, on fig. 4 , $b$, we compare only stabilised explicit methods of order one with the implicit Euler method. This experiment shows that damping is crucial for the general performance of a stabilised method. Another obvious conclusion is that for this particular problem the explicit methods are less accurate than the implicit one.

\section{Conclusion}

In this work we presented explicit multi-step methods of Adams type, which possess extended stability intervals. Simple formulae for the first order methods and their error constants are derived. We also applied damping to the first order methods, derived a general scheme for construction of stabilised $k$-step methods of any order $p<k$, and calculated coefficients for such methods numerically. It was shown that the error constant of the stabilised method grows as the number of steps increases, but this growth is quite slow. Our numerical experiments asserted the theoretical properties of accuracy and stability of the constructed methods and exhibited the importance of damping transformation for the methods.

In our opinion, at present the stabilised Adams-type methods are mostly of theoretical interest, but it cannot be ruled out that they could be useful in practice and become a basis for a competitive solver for mildly stiff problems. From a practical perspective the methods are attractive due to their low cost (just one evaluation of $f$ per step for any $k$ and $p$ ) and simplicity of implementation. The weak point is that long stability intervals require a large number of steps, which will entail memory issues, difficulties with the starting values and so on. 


\section{Mathematica code for computing the stabilised method's parameters}

Appendix A

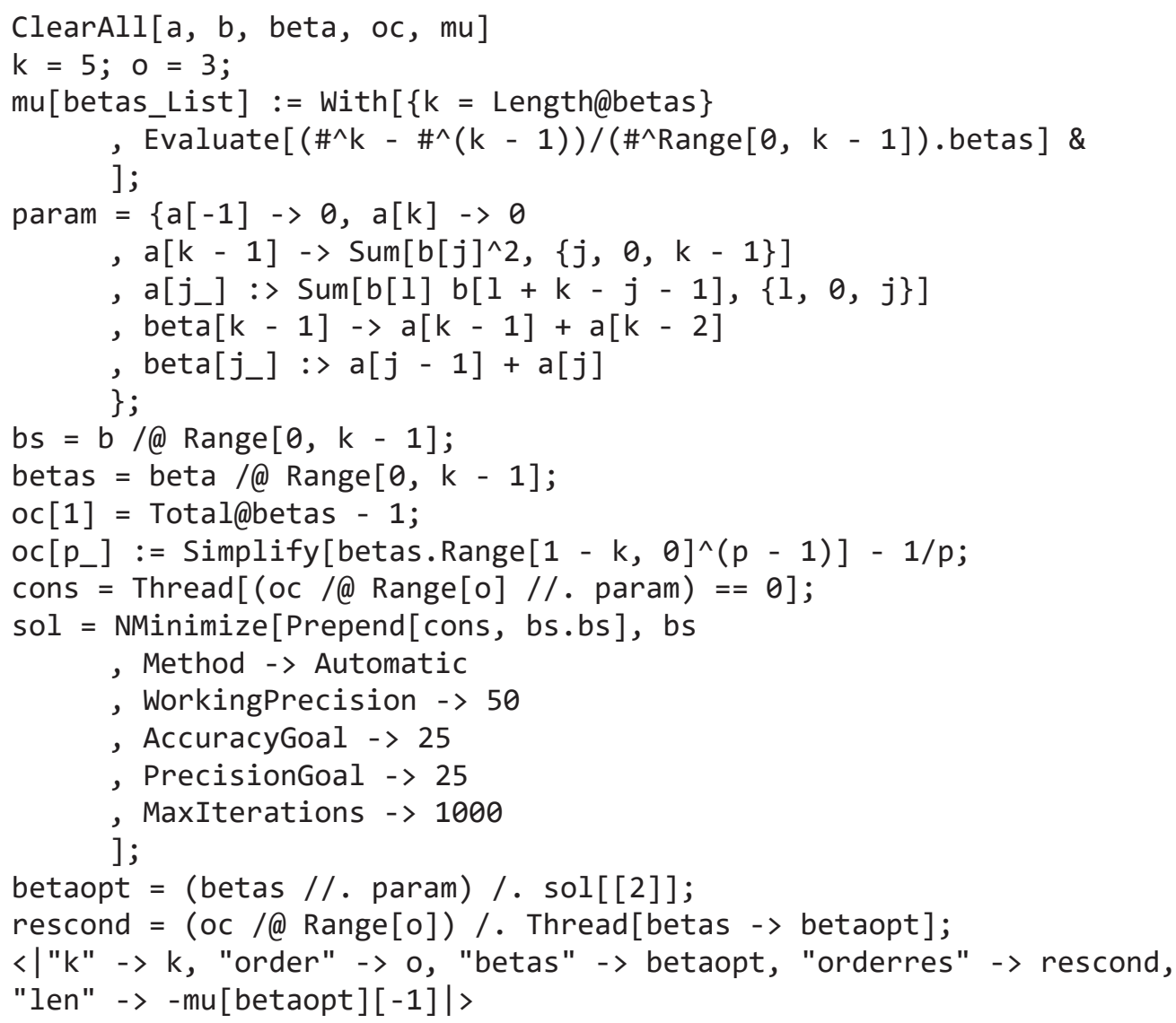

\section{References}

1. Hairer E, Wanner G. Solving ordinary differential equations II: stiff and differential-algebraic problems. Berlin: Springer; 1996. 614 p. (Springer series in computational mathematics; volume 14). DOI: 10.1007/978-3-642-05221-7.

2. Lebedev VI. How to solve stiff systems of differential equations by explicit methods. In: Marchuk GI, editor. Numerical methods and applications. Boca Raton: CRC Press; 1994. p. 45-80.

3. Sommeijer BP, Shampine LF, Verwer JG. RKC: an explicit solver for parabolic PDEs. Journal of Computational and Applied Mathematics. 1998;88(2):315-326. DOI: 10.1016/S0377-0427(97)00219-7.

4. Abdulle A, Medovikov AA. Second order Chebyshev methods based on orthogonal polynomials. Numerische Mathematik. 2001;90(1):1-18. DOI: 10.1007/s002110100292.

5. Abdulle A. Fourth order Chebyshev methods with recurrence relation. SIAM Journal on Scientific Computing. 2002;23(6): 2041-2054. DOI: 10.1137/S1064827500379549.

6. Jeltsch R, Nevanlinna O. Stability of explicit time discretizations for solving initial value problems. Numerische Mathematik. 1981;37(1):61-91. DOI: 10.1007/BF01396187.

7. Jeltsch R, Nevanlinna O. Stability and accuracy of time discretizations for initial value problems. Numerische Mathematik. 1982;40(2):245-296. DOI: 10.1007/BF01400542.

8. Daubechies I. Ten lectures on wavelets. Philadelphia: Society for Industrial and Applied Mathematics; 1992. 369 p. (CBMS-NSF regional conference series in applied mathematics).

9. Hairer E, Nørsett SP, Wanner G. Solving ordinary differential equations I: nonstiff problems. $2^{\text {nd }}$ edition. Berlin: Springer; 1993. 528 p. (Springer series in computational mathematics; volume 8). DOI: 10.1007/978-3-540-78862-1.

10. Xu Y, Zhao JJ. Estimation of longest stability interval for a kind of explicit linear multistep methods. Discrete Dynamics in Nature and Society. 2010;2010:1-18. DOI: 10.1155/2010/912691. 